\title{
Processing PET/CT Medicine Images and Determining the Object
}

\author{
Eminnur Topsakal Ayhan (Corresponding author) \\ Karadeniz Technical University, \\ Faculty of Engineering Geomatics Engineering, 61080 Trabzon-Turkey \\ E-mail: ayhan.eminnur@gmail.com \\ Salim Sercan Din \\ Karadeniz Technical University, \\ Faculty of Engineering Geomatics Engineering, 61080 Trabzon-Turkey \\ E-mail: salimsercandin@gmail.com
}

\begin{abstract}
Today, as a result of the developments in medical imaging systems, combined Positron Emission Tomography and Computed Tomography (PET/CT) images are used as effective imaging tools in cancer diagnosis and treatment. The study aims to display the tumor in 3D using PET/CT images and to determine the change in tumor size over time. For rapid detection of tumor tissues from numerous PET/CT images is developed software (in Phyton). With the software, tomography images taken at 3 different times 30.05.2017, 23.10.2017 and 08.02.2019 of a patient with hypopharynx cancer are evaluated. The image and real object coordinates of the cancerous region with a radioactive substance in the images are calculated. For 3 different times, variables related to changes in the tumor size of the patient are evaluated in Matlab software and 3D imaging and volume calculations are made. Thus, the temporal variation of the shape, location, and dimensions of the tumor is determined, and whether the patient's response to treatment is positive is observed with image processing and evaluation techniques.
\end{abstract}

Keywords: Medical İmage Processing, PET/CT imaging, Segmentation, Thresholding, Volume Rendering, Classification.

DOI: $10.7176 /$ JSTR/7-07-04

\section{Introduction}

The analysis of patient data acquired by current image modalities, such as Computerized Tomography (CT), Magnetic Resonance Tomography (MRT), Positron Emission Tomography (PET), or Ultrasound (US), offers previously unattained opportunities for diagnosis, therapy planning, and therapy assessment [1]. PET images have the potential to provide functional or biochemical information by measuring the distribution and kinetics of radioactivated molecules, while CT visualizes the X-ray intensity in tissues in the body [2]. Combined CT anatomical and PET functional imaging provides a stronger approach than PET and CT, which are made separately to monitor or target therapy [3]. FDG (18F-fluorodeoxyglucose) PET/CT images provide relevant information in staging and treatment monitoring of many tumors, including esophageal cancer, lung cancer, colorectal cancer, lymphoma, melanoma, and others [4].

In the literature, different image processing algorithms are used for different medical images. In the study comparing lung tumor segmentation methods in PET images, various image processing approaches were used to define the smoothness, roughness, and regularity of the involvement of intratumor FDG. Segmentation approaches have been applied after selecting a common/general region of interest (ROI) for all PET image sections transferred to the Matlab program. As a result of each approach, three-dimensional volume information and images were obtained in different patients and sections [5]. In the study of M. Sasikala et al., the region of interest detection algorithms for the

21 | P a g e

www.iiste.org 
detection of tumors in brain images are presented and compared. Gray level thresholding and wavelet transform methods were used to reveal abnormal structures. An artificial neural network was applied to the classification process [6]. In the study using brain MR sections, a method was developed for the detection of brain tumors with high accuracy, and volume calculation [7]. In their work, Xu and his friends made improvements to the images and performed a segmentation process based on pixel color density differences [8]. In the segmentation study in which PET images are used together with CT images, it was examined how tumor size was obtained more clearly and how it spread to other neighboring tissues [9].

In the study, PET/CT images belonging to a patient's relative are evaluated by image processing based on thresholding method. Numerical values of the dimensions of the tumor at 3 different times are obtained. The results are compared with the results of the doctor's report.

\section{Methods}

\section{Images Processing Techniques}

The most important stage of the treatment phenomenon is the diagnosis of the disease. The process of separating cancerous tissues from healthy tissues is determined with the help of image processing techniques, especially in the surgical procedures or treatments of cancer patients. One of the most common methods is to reveal the limit values depending on the contrast threshold value.

Image segmentation is the process of dividing the image into different areas where each area is homogeneous but the combinations of the two nearby areas are not homogeneous. It deals with medical image segmentation, visualization of anatomical parts, detection of abnormalities, measurement of tissues, preprocessing for surface registration, preprocessing for image registration, and their classification [10].

Images obtained with any medical imaging system are subjected to segmentation to examine certain organs. As a result of the segmentation process, the user is presented with volumetric and visual information [11]. Edge-based, field-based, fuzzy logic-based techniques, or artificial neural networks can be used for segmentation.

\section{Image Segmentation Methods}

Most of the segmentation algorithms are based on discontinuity and similarity, which are two basic principles of density values. The main purposes of segmentation algorithms are to divide the image based on the sudden intensity changes available at the object edges and to separate the image into areas according to predetermined similarity criteria. The point, line, and edge determination methods are based on the discontinuity principle of density values; thresholding, field widening, field dividing, and combining methods are based on the similarity principle [12]. Thanks to segmentation methods in image processing, the region of interest (ROI) can be removed and the tissues can be separated from each other [13]. In the PET/CT images in the study, the cancerous region appears bright due to the radioactive element it contains. Separation of the tumor from healthy tissues carried out by segmentation method.

The point, Line, Edge Determination: While the image is softened by the integral process used in the local average method, sudden local changes in density can be detected using the derivative process. Object boundaries are determined by taking first and second-order derivatives. The derivative of the numerical function is equivalent to making a difference. While the first-degree derivative process generally produces thick edges, the second-degree derivative process provides detailed information such as fine edges, isolated spots, and noise. By looking at the sign of the second-degree derivative, it can be determined that the transition from the determined border is from dark to light or from light to dark [12].

Thresholding: One of the simplest ways to segmentation an image. It is also used to convert grayscale images into binary images. If the gray level of objects of interest in the image is known or detected with the help of histograms, the image can be thresholded to highlight the detail of interest and destroy any remaining details [13].

Especially in many of the biomedical images, brightness is a distinctive feature in terms of

22 | P a g e

www.iiste.org 
segmentation and it is an application area where thresholding can be used [14].

Classification Methods: Classification is a pattern recognition technique performed with the help of a training set. The training data set is composed of examples with the characteristic features of the image. Classification is also called supervised learning since non-automated segmentation is made with training data and is then submitted to the automated process. The nearest-k-neighborhood, which is one of the simple classification methods, and the maximum likelihood method, which are among the parametric classification methods, are widely used classification methods [13, 15].

Clustering Methods: Clustering algorithms that do not use a training data set are unsupervised methods that have similar functioning with classification algorithms. In clustering algorithms, segmentation and extraction of the characteristic features of the classes are repeated alternately, thus eliminating the need for the training set [16].

\section{Volume Rendering}

When converting 3D information into 2D images, the most important point is not to lose too much volumetric information. The numerical triple point group, which is the smallest part of $2 \mathrm{D}$ images, is called a pixel, the concept of the pixel is voxel in 3D imaging. To achieve 3D modeling in the best way, the dimensions of voxels should be isotropic, that is, their coordinates on each third axis should be equal [17].

Volume is the clustered array of edited data points of 3D grids (computer square clusters). Grids can be regular, linear, curved, block-structured, or a mixture of these. In the medical field, regular grids are widely used, because high-resolution data is obtained on the XY plane in computed tomography while lower-resolution data is obtained on the $\mathrm{Z}$ plane. Volume creation includes all gray levels obtained during the scanning process. Unlike creating a surface; It does not require applying to geometry, it enables the display of weak surfaces. Volume calculation can be done in 2 ways;

- Feedback reflection or sequential image transition: each pixel in the image plane is passed over the volume with virtual irradiation, and the path followed by the rays determines the value of the pixels,

- Forward reflection or sequential object transition: the volume information is passed through and each voxel on the volume is projected onto the image plane [18].

\section{Data Used and Data Preparation}

PET/CT images, it is a proven Nuclear Medicine imaging technique that shows changes in the functions and metabolism of organs and tissues together with anatomical details. FDG-PET images show an increase in glycolysis in malignant cells compared to normal tissues. The most important feature of PET images and their main difference from radiological methods is that they are oriented to show functional and metabolic activity and do not show anatomical details. CT is used in the same imaging device to give anatomical information to PET images and is called PET/CT [5]. Especially in oncology, it is the most advanced medical imaging technique used for tumor detection, staging, evaluation of response to treatment, restaging in the case of metastasis, radiotherapy planning, and in some cases, whether the mass present is benign or malignant. Three-dimensional imaging of pathological areas showing normal and abnormal metabolic functions in organs and tissues is provided [19].

The PET/CT images used in the study belong to a relative of ours. These images used have the structure of a $284 \times 333$ pixels written on a DVD in DICOM format. With the help of the "Micro Dicom Viewer" program, PET/CT images are saved in 3 subfolders: Fused Coronal, Fused Sagittal, and Fused Transverse in jpeg format. Fused Coronal images include body from front to back, Fused Sagittal images include body from left to right, and Fused Transverse images include sections of the whole body scanned from the feet to the head, $3 \mathrm{~mm}$ apart. In the study, Fused Transverse images are used, where the cancerous area is seen and the readability of the data is good. PET/CT images include the type and date of shooting in the upper left, image slice and sequence numbers in the lower left, and the width and quality level of the image in the lower right (Fig. 1).

23 | P a g e www.iiste.org 


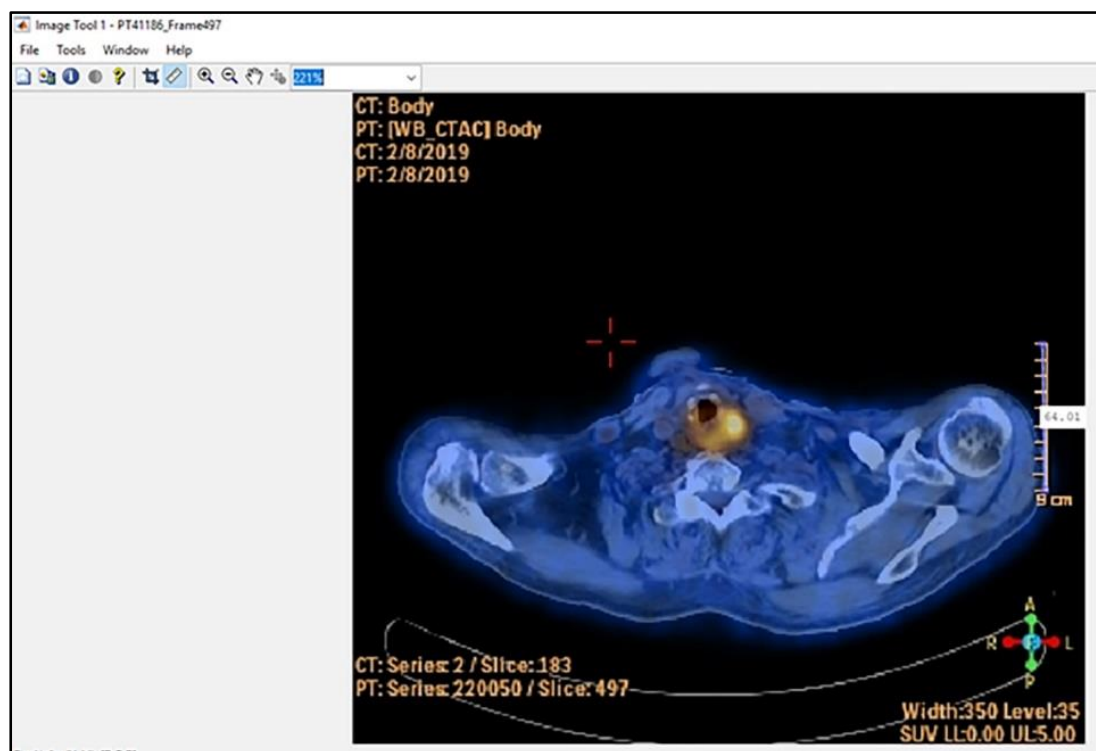

Fig.1 Cancerous area (bright) in PET/CT image, scale chart

While real 3 dimensions are obtained in the selected images, pixel values for $\mathrm{x}, \mathrm{y}$ axes are calculated with the help of the scale in the image with the Matlab "imtool" command, and z-axis is taken as $3 \mathrm{~mm}$ as specified in the shooting technique. In the PET/CT images, the cancerous area looks bright due to the radioactive element it contains. Thanks to, the values of the sample cancerous region $(\mathrm{R}, \mathrm{G}, \mathrm{B})$ are selected with the "pixel region" tool at Matlab. These processes are applied separately for PET/CT DVDs taken on 30.05.2017, 23.10.2017, and 08.02.2019, separate folders are created for each date, and the values are recorded.

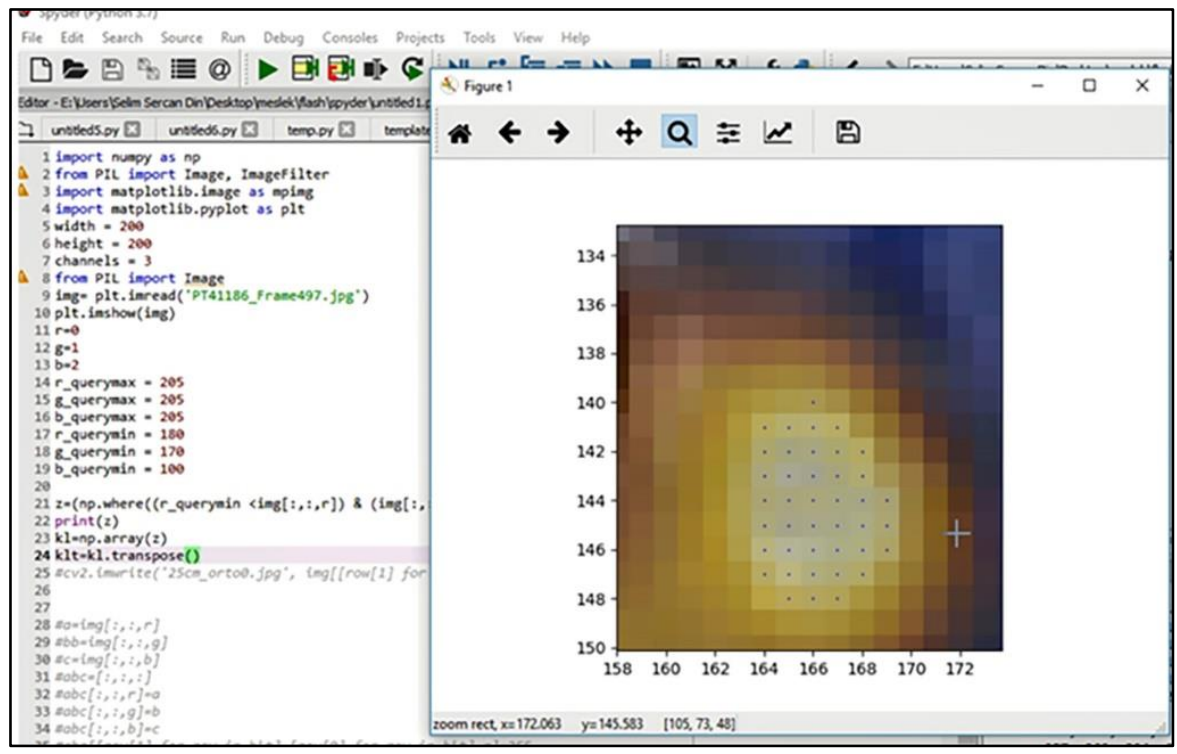

Fig. 2 Assignment of the R, G, B threshold values of the cancerous region in the algorithm

Algorithm Operation Steps: For the examination of the individual images, PET/CT images are recorded to different variables, and the sample " $R, G, B$ " values of the cancerous region obtained at the data preparation stage are assigned to the maximum and minimum variables. With Python 'where' command, all pixels in the images are compared with the defined "R, G, B" values (Fig. 2). If the pixel value is within the specified range, it is saved in temporary memory, if not, the next pixel is moved and 
operations are repeated. After all the pixels in the image are compared, the values stored in the temporary memory are recorded as row number $\mathrm{x}$ value and column number $\mathrm{y}$ value. The $\mathrm{z}$-axis is processed at $3 \mathrm{~mm}$ intervals as specified in the shooting technique. The coordinates of the cancerous regions in each image are combined with the help of the Python "zp.consantante" function. All operations are applied to approximately 160 images for shots of different dates. To reach the real values, calculated $\mathrm{x}, \mathrm{y}$, and $\mathrm{z}$ values are processed with the scale factor $9 \mathrm{~cm} / 64 \mathrm{px}$. The $\mathrm{x}, \mathrm{y}, \mathrm{z}$ values calculated on the images are recorded for 3D viewing in the Matlab environment (Fig. 3).

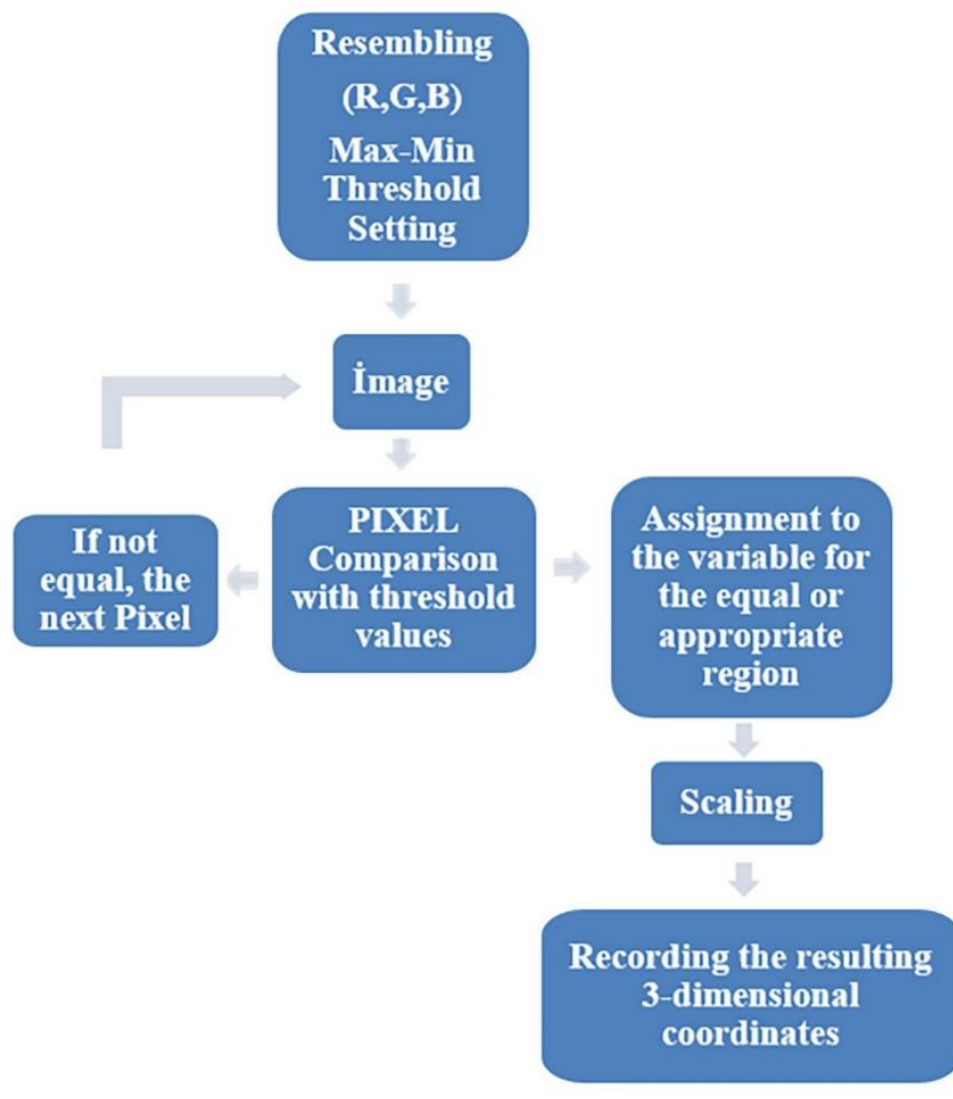

Fig. 3 Algorithm processing steps

\section{Discussion}

To date, there are numerous studies of processing and evaluation of medical images conducted by different disciplines. In this study, it is aimed to determine the change in time-dependent tumor sizes in 3D with the PET/CT images. With the developed software, the temporal status comparisons of the $x, y$, $\mathrm{z}$ dimensions of the tumor are made and the results about the course of the disease are revealed.

Thanks to the segmentation methods in image processing, the region of interest (ROI) can be removed and the tissues can be separated from each other. Expression of cerebrospinal fluid, white matter and gray matter in different colors on brain MR images; The separation of the tumor and edematous area from healthy tissues is achieved by segmentation methods (Norouzi et al. 2014). PET/CT images used in this study have different color density values (RGB) due to the radioactive element contained in the cancerous region. The region of interest (ROI) is subtracted from the images. A large number of images are scanned pixel by pixel with the software developed and the tumor is rapidly separated from healthy tissues by segmentation method. In study (Ogretmenoglu 2015), performed tumor segmentation by applying C-mean clustering and automatic threshing methods to sections with tumors in brain MRI images. Area calculations and volume calculations are made in segmented sections. In our study, tumor tissues in each section in PET / CT images are extracted based on color intensity threshold values with 
the software developed (Phyton Anaconda-Spyder interface). Areas and volumes are calculated with the position information of the pixels determined automatically from the image sections. Changes in tumor size are determined over time and 3-dimensional presentations are made. "Python" encoding is used for scanning and comparison calculations thanks to its high mathematical processing power and speed. Thanks to the ease and speed of the 3D display algorithm, Matlab coding is used in demonstrating the result data.

For the test, the tumor determined by the specialist doctors is compared with the tumor determined automatically by image processing method. For this purpose, the doctor's reports and the values obtained from the evaluation of the images are compared. As a result of the report dated 30.05.2017, it was stated that minimal concentric wall thickness increase was observed in the proximal part of the esophagus, the patient was followed up due to the hypopharyngeal tumor and chemotherapy and radiotherapy were given. As a result of the 23.10.2017 report, it was reported that the tumor seen in the esophagus decreased significantly compared to the previous PET/CT examinations. As a result of the report dated 08.02.2019, it was emphasized that when compared to the previous PET/CT examination, it was spread from the distal oropharynx and proximal $1 / 3$ section of the esophagus to the laryngeal cartilage, extending in the hypopharynx and the laryngeal cartilage.

The results obtained by processing PET/CT images are in full agreement with patient reports. Approximate tumor sizes are calculated as $3 \mathrm{~cm}$ for $\mathrm{x}$ and $\mathrm{y}, 9 \mathrm{~cm}$ for $\mathrm{z}$ from images dated 30.05.2017, and $0.3 \mathrm{~cm}$ for $\mathrm{x}$ and $\mathrm{y}$ and $1 \mathrm{~cm}$ for $\mathrm{z}$ from images dated 23.10.2017. It was also determined by the patient report that the patient's responded positively to the treatment. Therefore, radiotherapy treatment was terminated because the patient was considered to have recovered. However, in the image evaluation dated 08.02.2019 taken in the last controls, it is calculated that the tumor sizes reached approximately $1 \mathrm{~cm}$ in the $\mathrm{x}$ and $\mathrm{y}$ and $7 \mathrm{~cm}$ in the $\mathrm{z}$. For images taken at different times, the $\mathrm{x}, \mathrm{y}, \mathrm{z}$ values of the pixels of the cancerous region and the dimensions of the tumor in the $x y, x z$ and $y z$ axes are also shown in Fig. $\underline{4}$.

Tumor volume calculation is made by Matlab solid waste modeling. With this study, tumor volume is calculated as $28 \mathrm{~cm} 3$ in the image dated 30.05.2017, $0.016 \mathrm{~cm} 3$ in the image dated 23.10.2017, and $1.92 \mathrm{~cm} 3$ in the last image dated 08.02.2019 (Fig. 5). Consistent with these results, it was seen in the patient reports that the patient was offered chemotherapy again.
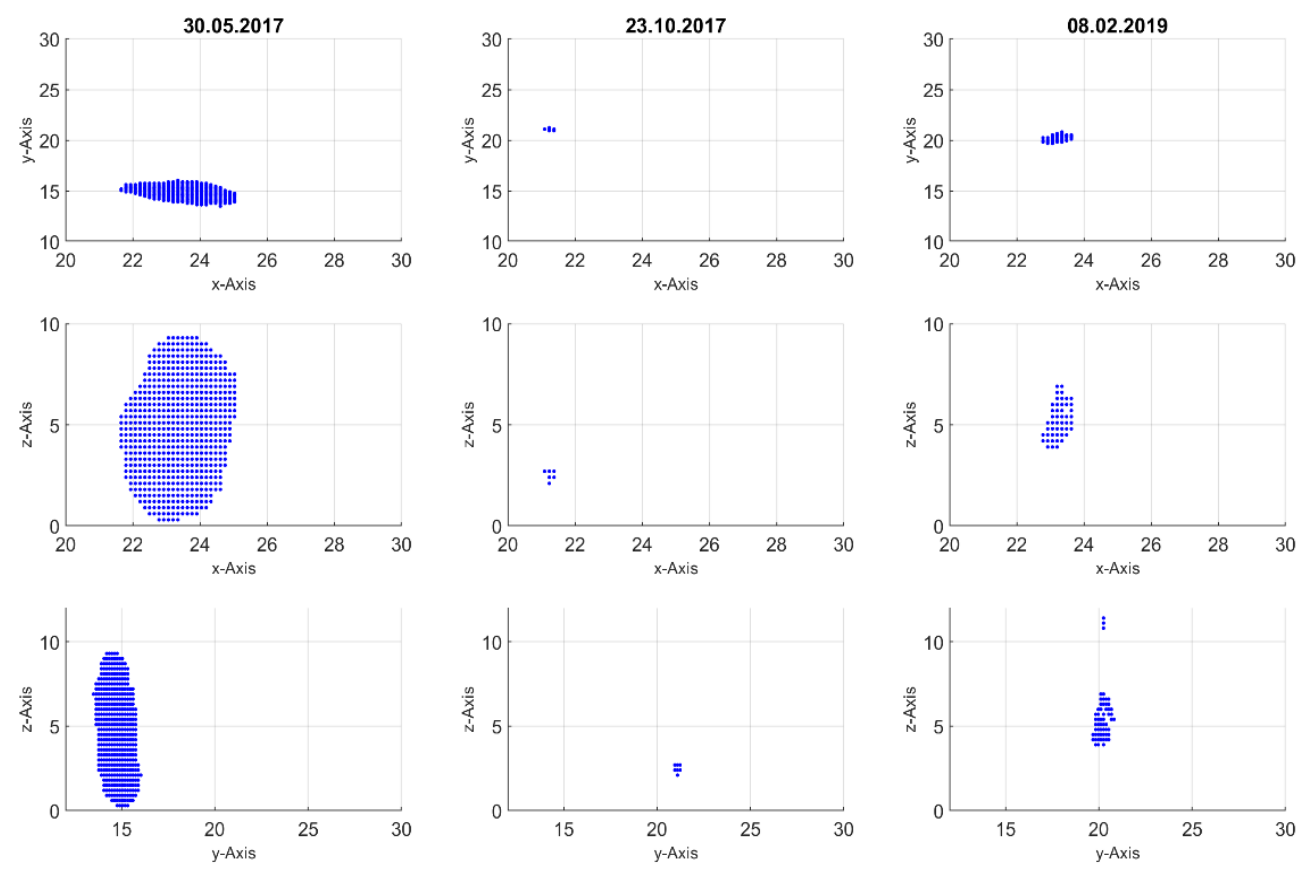

Fig. 4 Tumor position and temporal changes in xy, xz, and yz axes respectively

26 I P a g e

www.iiste.org 


\author{
Vol.
}
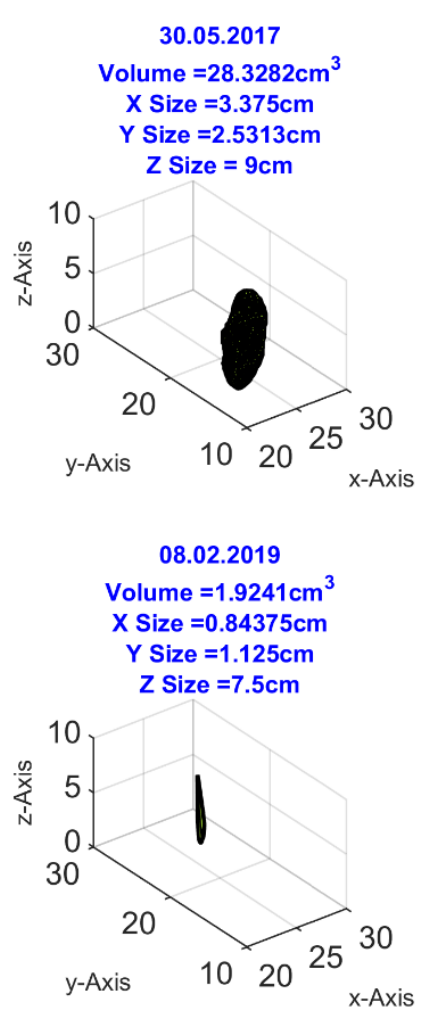

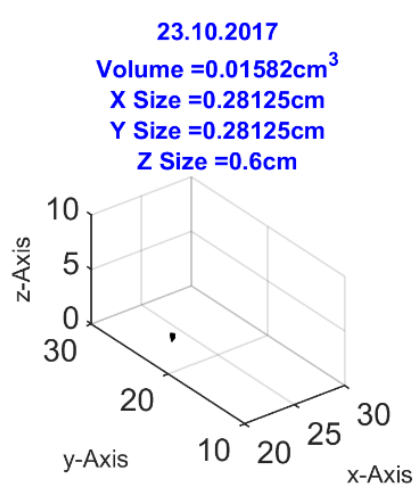

23.10.2017

$=0.28125 \mathrm{~cm}$

ize $=0.28125 \mathrm{~cm}$

Fig. 5 Tumor locations and volumes for PET/CT images taken on 3 different dates

\section{Conclusions}

In the study, which aimed to obtain tumor sizes from PET/CT images taken at different times, firstly, samples are taken from the radioactive region and recorded. The pixels of all the images are compared with the samples, and the image coordinates of the tumor are calculated by Python programming and the actual dimensions are obtained with the help of the image scale chart.

From image evaluation results;

In the image dated 30.05 .2017 , it is observed that the tumor volume was $28.3282 \mathrm{~cm} 3$ and $3.3750 \mathrm{~cm}$, $2.5313 \mathrm{~cm}$, and $9.0 \mathrm{~cm}$ in the $\mathrm{x}, \mathrm{y}$, and $\mathrm{z}-$ axes respectively.

In the image dated 23.10.2017, approximately 5 months later, it is observed that the tumor volume decreased to $0.01582 \mathrm{~cm} 3$, and the dimensions in the $\mathrm{x}, \mathrm{y}$, and $\mathrm{z}$ axes decreased to $0.28125 \mathrm{~cm}$, $0.28125 \mathrm{~cm}$ and $0.6 \mathrm{~cm}$, respectively. As a result of the evaluations, it is revealed that there is a noticeable difference between the tumor sizes five months ago and that the patient responded positively to the treatment.

The tumor volume is calculated as $1.9241 \mathrm{~cm} 3$ for the image dated 08.02 .2019 and it is determined that the $\mathrm{x}, \mathrm{y}$, and $\mathrm{z}$-axis dimensions were $0.84375 \mathrm{~cm}, 1.125 \mathrm{~cm}, 7.5 \mathrm{~cm}$, respectively. In approximately 1 year and 3 months between the last imaging and the previous imaging, it is determined that the patient had another tumor in a different position both numerically and visually.

The importance of tumor detection and 3-dimensional imaging from medical images during the diagnosis, preparation of treatment plans, and treatment stages have been demonstrated by studies conducted to date. With this study, using the developing software and techniques, rapid detection of tumor tissues from many PET/CT images (approximately 160 images), real size measurements, and 3D imaging are performed. Advances in imaging types in the field of Medicine are ongoing. Parallel to developments, different methods and very different disciplines continue to work for the processing, evaluation of images and the extraction of desired information sensitively. 


\section{Compliance with ethical standards}

Conflict of interest On behalf of all authors, the corresponding author states that there is no conflict of interest.

\section{References}

1. Ritter F, Boskamp T, Homeyer A, Laue H, Schwier M, Link F, and Peitgen H-O (2011) Medical Image Analysis, IEEE Plus, Nov;2(6):60-70. doi: 10.1109/MPUL.2011.942929 PubMed ID: 22147070

2. Razifar P, Sandström M, Schnieder H, Långström B, Maripuu E, Bengtsson E, Bergström M (2005) Noise correlation in PET, CT, SPECT and PET/CT data evaluated using autocorrelation function: a phantom study on data, reconstructed using FBP and OSEM, BMC Medical Imaging, 5(1):5. doi: 10.1186/1471-2342-5-5, Corpus ID: 2898490

3. Fukui M B, Blodgett T M, Snyderman C H, Johnson J J, Myers E N, Townsend D W, Meltzer C C (2005) Combined PET-CT in the Head and Neck Part 2. Diagnostic Uses and Pitfalls of Oncologic Imaging1. Published Online: 01 Jul 2005, https://doi.org/10.1148/rg.254045136

4. Chen Y-K, Ding H-J, Su C-T, Shen Y-Y, Chen L-K, Liao A C, Hung T-Z, Hu F-L, and Kao C-H (2004) Application of PET and PET/CT Imaging for Cancer Screening, Anticancer Research 24: 4103-4108, PMID: 15736459

5. Eset K, İçer S, Karaçavuş S, Yılmaz B, Kayaaltı Ö, Ayyıldız O, Kaya E (2015) Comparison of Lung Tumor Segmentation Methods on PET Images, Medical Technologies National Conference (TIPTEKNO), IEEE, 978-1-4673-7765-2, 2015, doi: 10.1109/ TIP TEKNO.2015.7374569

6. Sasikala M, Kumaravel N (2005) Comparison of Feature Selection Techniques for Detection of Malignant Tumor in Brain Images. IEEE Indicon 2005 Conference11-13 Dec., Chennai, India, doi: 10.1109/INDCON.2005.1590157

7. Ogretmenoglu C, Erogul O, Telatar Z, Guler R E and Yildirim F (2015) Brain Tumor Detection and Volume Estimation via MR imaging, Journal of Biotechnology, 208, S15, https://doi.org/10.1016/j.jbiotec.2015.06.033

8. Xu L, et al. (1999) Segmentation of skin cancer images, Image and Vision Computing 17, 65-74, https://doi.org/10.1016/S0262-8856(98)00091-2

9. Ballangan C, Wang X, Fulham M, Eberl S and Feng D D (2013) Lung tumor segmentation in PET images using graph cuts, Computer Methods, and Programs in Biomedicine, 109, vol 3, 260-268, doi: 10.1016/j.cmpb.2012.10.009, PMID: 23146420

10. Kim D S, Huang H, Dhawan K, Atam P (2008) Principles and Advanced Methods in Medical Imaging and Image Analysis, Singapore: World Scientific, https://doi.org/10.1142/6366

11. Singh H, Crawford M, Curtin J and Zwiggelaar M (2004) Automated 3D segmentation of the lung airway tree using gain-based region growing approach, Medical Image Computing and Computer-Assisted Intervention - MICCAI, vol 2, 975-982, Automated 3D Segmentation of the Lung Airway Tree Using Gain-Based Region Growing Approach | SpringerLink

12. Gonzalez, R. C. and Woods, R. (2010) Digital image processing. 3rd Edition, Pearson Prentice Hall, New Jersey. 
13. Norouzi A, Rahim M, Altameem A, Saba T, Rad A, Rehman A and Uddin M (2014) Medical Image Segmentation Methods, Algorithms and Applications. IETE Technical Review, 31(3), 199-213, https://doi.org/10.1080/02564602.2014.906861

14. Sezgin M, Taşaltın R (2000) A New dichotomization technique to multilevel thresholding devoted to inspection applications, Pattern Recognition Letters, 21, 151-161, https://doi.org/10.1016/S0167-8655(99)00142-7

15. Ayhan E, Kansu O (2012) Analysis of Image Classification Methods for Remote Sensing, Experimental Techniques, Vol. 36, 18-25, https://doi.org/10.1111/j.1747-1567.2011.00719.x

16. Pham D, Xu C and Prince J (2000) Current Methods in Medical Image Segmentation. Annual Review of Biomedical Engineering, 2, 315-337, https://doi.org/10.1146/annurev.bioeng.2.1.315

17. Remondino F (2003) from point cloud to surface: the modeling and visualization problem, International Workshop on Visualization and Animation of Reality-based 3D Models, 26, Tarasp-Vulpera, Switzerland, https://doi.org/10.3929/ethz-a-004655782

18. Baert A, Knauth M, Sartor K, eds. (2007) Medical Radiology Diagnostic Imaging, p: 31-35

19.https://hastaneler.erciyes.edu.tr/Eskihaberler/petbt/PET-BT\%20A\%C3\%87ILI\% C5\% 9E $\%$ 20T\%C3\%96REN\%C4\%B0.pdf, 06 May 2019. 